\title{
Influence of Pressure on Magnetic Properties of $\mathrm{La}_{1-x} \mathrm{Sr}_{x} \mathrm{CoO}_{3-\delta}(x=0.5,0.67,1)$
}

\author{
J. Piestosa ${ }^{a}$, A. Wiśniewski ${ }^{a}$, R. Puźniak ${ }^{a}$, S. Kolesnik $^{b}$, \\ M. MAJJIGA ${ }^{b}$ AND B. DABROWSKI ${ }^{b}$ \\ ${ }^{a}$ Institute of Physics, Polish Academy of Sciences \\ al. Lotników 32/46, 02-668 Warsaw, Poland \\ ${ }^{b}$ Physics Department, Northern Illinois University, De Kalb, IL 60115, USA
}

Influence of pressure on the phase transition temperature $T_{\mathrm{C}}$ and on the spontaneous magnetisation $M_{0}$ of highly Sr substituted and oxygen nonstoichiometric $\mathrm{La}_{1-x} \mathrm{Sr}_{x} \mathrm{CoO}_{3-\delta}$ samples was investigated. An increase in $T_{\mathrm{C}}$ and $M_{0}$ with pressure in investigated samples was found, indicating strengthening of ferromagnetic double exchange interactions, and the expansion of volume of ferromagnetic phase as a result of enhancement in ferromagnetic interactions in both cluster-glass and long range ferromagnetic states. We did not observe clear correlation between changes in pressure coefficient $\mathrm{d} T_{\mathrm{C}} / \mathrm{d} P$ and changes in the formal oxidation state of the Co ion.

PACS numbers: 75.30.-m, 75.47.Gk, 75.30.Kz

\section{Introduction}

$\mathrm{La}_{1-x} \mathrm{Sr}_{x} \mathrm{CoO}_{3-\delta}$ solid solution oxides crystallize in perovskite-type $\mathrm{ABO}_{3}$ structure with a lanthanide ion (e.g. $\mathrm{La}^{3+}$ ) or an alkaline earth ion $\left(\right.$ e.g. $\mathrm{Sr}^{2+}$ ) at the A site and with a cobalt ion at the B site. These oxides with $x=0-0.5$ have been recently intensively studied due to their interesting transport and magnetic properties, for example metal-insulator transition, which sensitively depend on $\mathrm{Sr}$ content [1]. An additional feature that results from the oxygen vacancies is the so-called mixed conductivity, which is a combination of high ionic conductivity and high electronic conductivity [2]. It opens possibilities for practical applications of these materials as high temperature ceramic membranes [3] and as cathode materials for solid oxide fuels [4].

Typically, the magnetic properties of $\mathrm{La}_{1-x} \mathrm{Sr}_{x} \mathrm{CoO}_{3-\delta}$ are affected by temperature and magnetic field. Another parameter, which enables us to control 
physical properties, is external pressure. It was shown that pressure might have

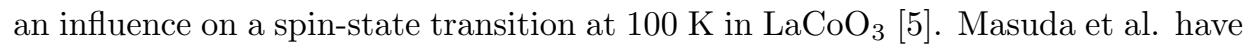
found resistivity anomaly at low temperature under pressure in $\operatorname{Pr}_{0.7} \mathrm{Ca}_{0.3} \mathrm{CoO}_{3}$ which indicated the existence of the phase transition [6]. In Ref. [7] it was found that external pressure causes the phase transition to occur from a nearly metallic to an insulating state in compound $\mathrm{Pr}_{0.8} \mathrm{Ca}_{0.2} \mathrm{CoO}_{3}$, in which normally, at ambient pressure such a transition is not observed. In conducting, ferromagnetic single crystal $\mathrm{La}_{0.82} \mathrm{Sr}_{0.18} \mathrm{CoO}_{3}$, a pressure-induced increase in electrical resistivity and decrease in the ferromagnetic transition temperature has been observed [8]. The pressure effect in low doped single crystals $\mathrm{La}_{1-x} \mathrm{Ca}_{x} \mathrm{CoO}_{3}$ and $\mathrm{La}_{1-x} \mathrm{Sr}_{x} \mathrm{CoO}_{3}$ was investigated by Fita et al. [9]. For Ca-doped material the applied pressure strongly suppressed the ferromagnetic (FM) interactions as well as volume of FM phase, while for Sr-doped compound it was shown that the pressure enhanced FM interactions at $x=0.3$ and suppressed at $x=0.2$.

In this paper, we present first results of magnetic measurements under pressure performed for highly Sr substituted $\mathrm{La}_{1-x} \mathrm{Sr}_{x} \mathrm{CoO}_{3-\delta}(x \geq 0.5)$ polycrystals with different oxygen contents.

\section{Experimental details}

The conventional solid state reaction was used to prepare single-phase polycrystalline samples of $\mathrm{La}_{0.5} \mathrm{Sr}_{0.5} \mathrm{CoO}_{2.89}, \mathrm{La}_{0.33} \mathrm{Sr}_{0.67} \mathrm{CoO}_{2.85}, \mathrm{SrCoO}_{2.88}$ and $\mathrm{SrCoO}_{2.67}$. Appropriate molar ratios of $\mathrm{La}_{2} \mathrm{O}_{3}, \mathrm{SrCoO}_{3}$ and $\mathrm{Co}_{3} \mathrm{O}_{4}$ were mixed and repetitively finely ground and fired in air at temperatures between 800 and $1000^{\circ} \mathrm{C}$. The samples were then pressed into pellets and fired at $1100-1120^{\circ} \mathrm{C}$. Obtaining single-phase sample of $\mathrm{SrCoO}_{x}$ required quenching from the high temperatures on copper plate. Samples with various oxygen content were obtained by additional annealing in high-pressure oxygen $\left(\mathrm{SrCoO}_{2.88}\right)$, or air followed by quenching in liquid nitrogen. Oxygen content of these samples was checked by thermogravimetric measurements. All the magnetic measurements were performed in the temperature range $4.2-250 \mathrm{~K}$ at magnetic field up to $16 \mathrm{kOe}$ using a PAR 4500 vibrating sample magnetometer. Temperature dependence of magnetisation was measured using zero-field-cooling (ZFC) and field-cooling (FC) procedure, whereas $M(H)$ dependence was measured after $\mathrm{FC}$ in maximum applied field. For these measurements a miniature container of $\mathrm{CuBe}$ [10] with an inside diameter of $1.42 \mathrm{~mm}$ was employed as a pressure cell. Mixtures of mineral oil-kerosene and mineral oil-silicone were used as a pressure transmitting mediums. The pressure at low temperature was determined by the pressure dependence of the superconducting transition temperature of pure tin placed near the sample.

\section{Results and discussion}

From the ZFC and FC magnetisation curves (Figs. 1a, 2a, 3a, 4a) it can be seen that the ferromagnetic transition temperature $T_{\mathrm{C}}$ increases with pressure 
for all samples. $T_{\mathrm{C}}$ was determined from the magnetisation $M(T)$ curves as the temperature of the maximum slope of $-\mathrm{d} M / \mathrm{d} T$. In Figs. $1 \mathrm{~b}, 2 \mathrm{~b}, 3 \mathrm{~b}, 4 \mathrm{~b}$ the FC hysteresis loops measured at $T=10 \mathrm{~K}$ are shown. All samples, except $\mathrm{SrCoO}_{2.67}$, show clear spontaneous FM moment $M_{0}$, which was extracted by linear extrapolation of $M(H)$ from high-field region to $H=0$. The existence of $M_{0}$ together with a marked difference between $M_{\mathrm{ZFC}}$ and $M_{\mathrm{FC}}$ at low temperatures indicates ferromagnetism below $T_{\mathrm{C}}$. The observed changes of $T_{\mathrm{C}}$ and $M_{0}$ can be approximated by linear pressure dependence with the pressure coefficients of $\mathrm{d} T_{\mathrm{C}} / \mathrm{d} P$ and $\mathrm{d} M_{0} / \mathrm{d} P$ for $T_{\mathrm{C}}$ and $M_{0}$, respectively. It is generally accepted that ferromagnetism in doped $\mathrm{La}_{1-x} \mathrm{Sr}_{x} \mathrm{CoO}_{3}$ arises as a result of the double exchange (DE) interactions between $\mathrm{Co}^{3+}$ and $\mathrm{Co}^{4+}$. Therefore, increase in $T_{\mathrm{C}}$ implies strengthening of FM DE interactions. The increase in $M_{0}$ indicates volume expansion of FM phase.
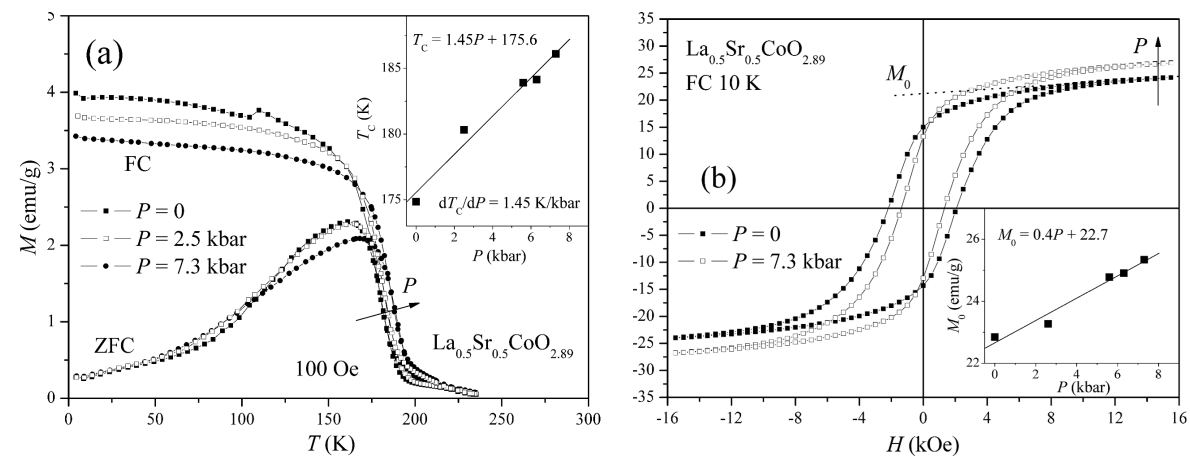

Fig. 1. (a) Temperature dependence of $M_{\mathrm{ZFC}}$ and $M_{\mathrm{FC}}$ for $\mathrm{La}_{0.5} \mathrm{Sr}_{0.5} \mathrm{CoO}_{2.89}$ measured at 100 Oe. Inset: pressure dependence of the Curie temperature. (b) Magnetisation hysteresis loops for $\mathrm{La}_{0.5} \mathrm{Sr}_{0.5} \mathrm{CoO}_{2.89}$ at $T=10 \mathrm{~K}$. Inset: pressure dependence of spontaneous magnetisation $M_{0}$.

The formal oxidation state of cobalt $\nu_{\mathrm{Co}}=3+x-2 \delta$ is +3.28 and +3.37 for $\mathrm{La}_{0.5} \mathrm{Sr}_{0.5} \mathrm{CoO}_{2.89}$ and $\mathrm{La}_{0.33} \mathrm{Sr}_{0.67} \mathrm{CoO}_{2.85}$, respectively. For these samples we observe qualitatively similar dependence of $M(T)$ and $M(H)$. The samples are typical ferromagnets with $\mathrm{DE}$ as a dominating interaction. For $\mathrm{La}_{0.33} \mathrm{Sr}_{0.67} \mathrm{CoO}_{2.85}$, the $M_{0}$ increases with pressure in a non-linear way (inset of Fig. 2b). For the $\mathrm{SrCoO}_{2.88}$ sample with the highest $\nu_{\mathrm{Co}}=+3.76$ the $M_{0}$ increases very slightly with pressure (Fig. $3 \mathrm{~b}$ ). It suggests very small expansion of FM phase volume. No increase in the FM phase volume is seen for the $\mathrm{SrCoO}_{2.67}\left(\nu_{\mathrm{Co}}=+3.34\right)$ sample with the highest amount of oxygen vacancies. In general, an increase in the pressure coefficient $\mathrm{d} T_{\mathrm{C}} / \mathrm{d} P$ with increasing $\nu_{\mathrm{Co}}$ is observed with an exception of the $\mathrm{SrCoO}_{2.67}$ sample for which it reaches the minimal value. From the $M(H)$ curve for $\mathrm{SrCoO}_{2.67}$ one can see drastic decrease in magnetisation when compared with the other samples (Fig. 4b). Strong dependence of $M$ on $H$ can be seen even in high magnetic field, which suggests that the FM interactions are 

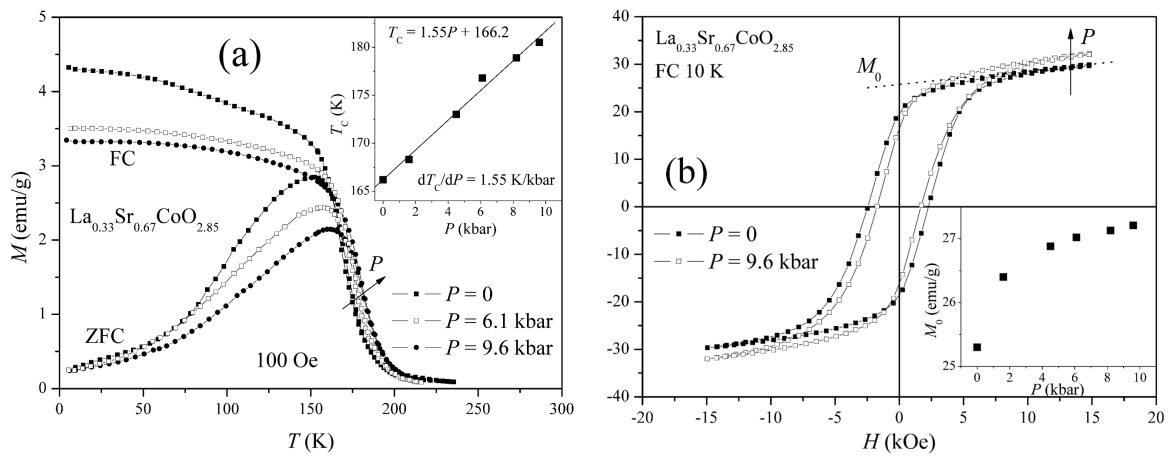

Fig. 2. (a) Temperature dependence of $M_{\mathrm{ZFC}}$ and $M_{\mathrm{FC}}$ for $\mathrm{La}_{0.33} \mathrm{Sr}_{0.67} \mathrm{CoO}_{2.85}$ measured at 100 Oe. Inset: pressure dependence of the Curie temperature. (b) Magnetisation hysteresis loops for $\mathrm{La}_{0.33} \mathrm{Sr}_{0.67} \mathrm{CoO}_{2.85}$ at $T=10 \mathrm{~K}$. Inset: pressure dependence of spontaneous magnetisation $M_{0}$.
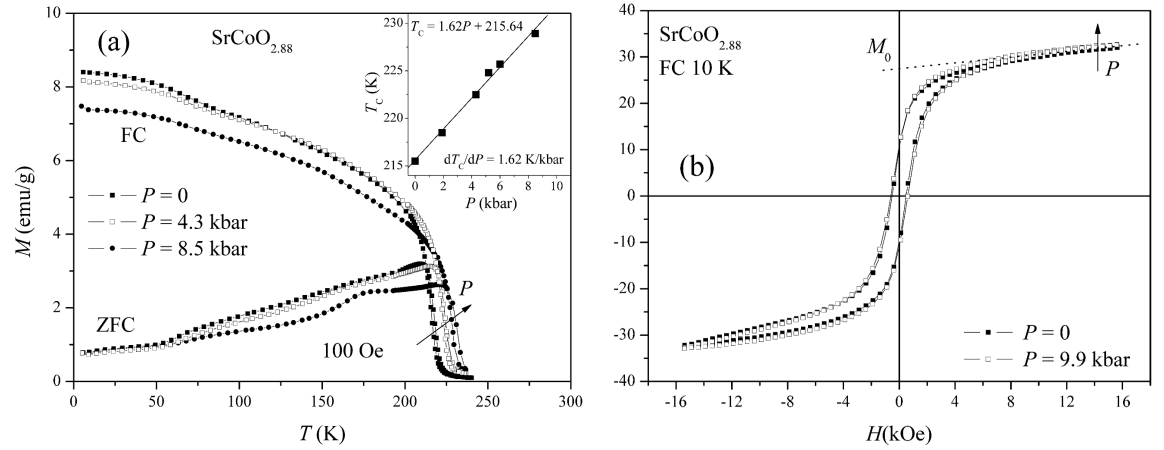

Fig. 3. (a) Temperature dependence of $M_{\mathrm{ZFC}}$ and $M_{\mathrm{FC}}$ for $\mathrm{SrCoO}_{2.88}$ measured at 100 Oe. Inset: pressure dependence of the Curie temperature. (b) Magnetisation hysteresis loops for $\mathrm{SrCoO}_{2.88}$ at $T=10 \mathrm{~K}$.

not dominating anymore. Similar behaviour has been observed for highly oxygen deficient $\mathrm{La}_{0.33} \mathrm{Sr}_{0.67} \mathrm{CoO}_{2.51}$ sample [11]. This behaviour has been attributed to weak nature of ferromagnetism, probably associated with canted antiferromagnetic state.

For the $\mathrm{La}_{1-x} \mathrm{Sr}_{x} \mathrm{CoO}_{3}$ system with low Sr-doping, it was reported by Fita et al. that the $\mathrm{d} T_{\mathrm{C}} / \mathrm{d} P$ equals to -0.28 and to $0.3 \mathrm{~K} / \mathrm{kbar}$ for $x=0.2\left(\nu_{\mathrm{Co}}=+3.2\right)$ and for $x=0.3\left(\nu_{\mathrm{Co}}=+3.3\right)$, respectively [9]. Clearly, the $x=0.2$ sample with a negative value of $\mathrm{d} T_{\mathrm{C}} / \mathrm{d} P$ behaves anomalously. It may be because the $\mathrm{La}_{0.8} \mathrm{Sr}_{0.2} \mathrm{CoO}_{3}$ sample is at percolation threshold (PT) where the minimal and negative value of $\mathrm{d} T_{\mathrm{C}} / \mathrm{d} P$ has been postulated [9]. The $\mathrm{La}_{0.5} \mathrm{Sr}_{0.5} \mathrm{CoO}_{2.89}$ sample studied here with a similar $\nu_{\mathrm{Co}}=+3.28$ may be above the PT. If we assume that the decrease in $\mathrm{d} T_{\mathrm{C}} / \mathrm{d} P$ denotes for approach to $\mathrm{PT}$ we would find PT close to $\nu_{\mathrm{Co}}=+3.34$ where the lowest $\mathrm{d} T_{\mathrm{C}} / \mathrm{d} P$ but not negative value is observed 

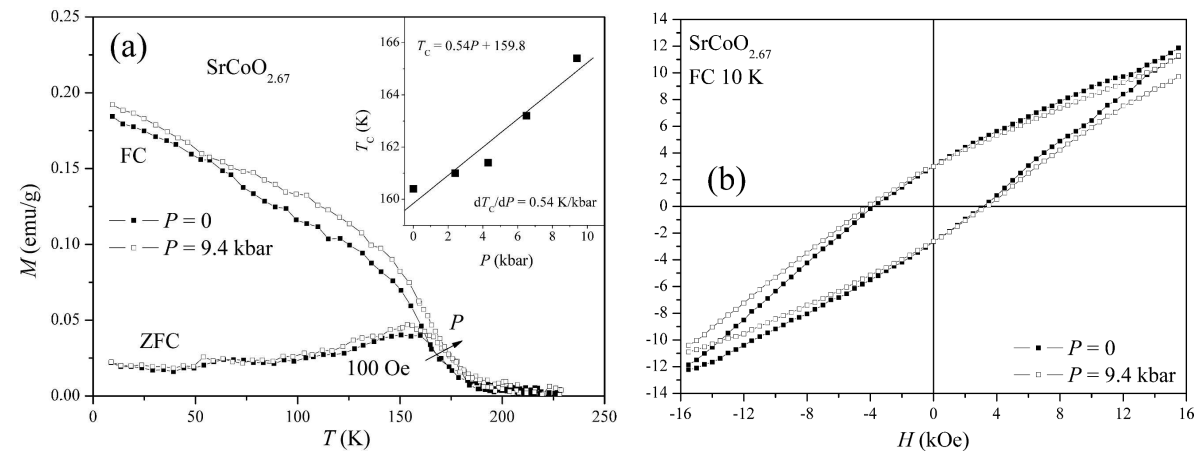

Fig. 4. (a) Temperature dependence of $M_{\mathrm{ZFC}}$ and $M_{\mathrm{FC}}$ for $\mathrm{SrCoO}_{2.67}$ measured at 100 Oe. Inset: pressure dependence of the Curie temperature. (b) External field dependence on magnetisation of $\mathrm{SrCoO}_{2.67}$ at $T=10 \mathrm{~K}$.

for samples not stoichiometric in oxygen content. The discrepancy between the $\mathrm{d} T_{\mathrm{C}} / \mathrm{d} P$ value for the nonstoichiometric sample with $\nu_{\mathrm{Co}}=+3.28$ and $\mathrm{d} T_{\mathrm{C}} / \mathrm{d} P$ values for the stoichiometric samples with $\nu_{\mathrm{Co}}=+3.2$ and $\nu_{\mathrm{Co}}=+3.3$ together with the lowest $\mathrm{d} T_{\mathrm{C}} / \mathrm{d} P$ value observed at $\nu_{\mathrm{Co}}=+3.34$ suggests that valence state of Co ion is not a key factor controlling magnetic properties. Other factors such as the oxygen content and its possible short or long-range ordering as well as cobalt spin state may be equally important. Further studies of magnetic properties are required for systems with different level of doping and with fixed oxygen vacancy content.

\section{Conclusions}

The influence of pressure on magnetic properties of highly Sr substituted $\mathrm{La}_{1-x} \mathrm{Sr}_{x} \mathrm{CoO}_{3-\delta}$ was investigated. We observed an increase in $T_{\mathrm{C}}$ and $M_{0}$ with pressure in all investigated samples, except $\mathrm{SrCoO}_{2.67}$ where the pressure effect on $M_{0}$ could not be seen. Enhancement of ferromagnetic properties was attributed to the strengthening of FM DE interactions, and the expansion of volume of $\mathrm{FM}$ phase. We suggest that $\mathrm{DE}$ interaction dominates in $\mathrm{La}_{0.5} \mathrm{Sr}_{0.5} \mathrm{CoO}_{2.89}, \mathrm{La}_{0.33} \mathrm{Sr}_{0.67} \mathrm{CoO}_{2.85}$ and $\mathrm{SrCoO}_{2.88}$. We did not observe clear correlation between changes in pressure coefficient $\mathrm{d} T_{\mathrm{C}} / \mathrm{d} P$ and changes in the formal oxidation state of Co ion.

\section{Acknowledgments}

This work was supported partly by the State Committee for Scientific Research, Poland (Project No. 1P 03B 038 27). Work at NIU was supported by the NSF-DMR-0302617 and by the U.S. Department of Transportation. 


\section{References}

[1] J. Wu, C. Leighton, Phys. Rev. B 67, 174408 (2003).

[2] Y. Teraoka, T. Nobunaga, K. Okamoto, N. Miura, N. Yamazoe, Solid State Ionics 48, 207 (1991).

[3] H.Y. Tu, Y. Takeda, N. Imanishi, O. Yamamoto, Solid State Ionics 100, 283 (1997).

[4] A.V. Kovalevsky, V.V. Kharton, V.N. Tikhonovich, E.N. Naumovich, A.A. Tonoyan, O.P. Reut, L.S. Boginsky, Mater. Sci. Eng. B 52, 105 (1998).

[5] K. Asai, O. Yokokura, M. Suzuki, T. Naka, T. Matsumoto, H. Takahashi, N. Mori, K. Kohn, J. Phys. Soc. Jpn. 66, 967 (1997).

[6] H. Masuda, T. Fujita, T. Miyashita, M. Soda, Y. Yasui, Y. Kobayashi, M. Sato, J. Phys. Soc. Jpn. 72, 873 (2003).

[7] T. Fujita, T. Miyashita, Y. Yasui, Y. Kobayashi, M. Sato, E. Nishibori, M. Sakata, Y. Shimojo, N. Igawa, Y. Ishii, K. Kakurai, T. Adachi, Y. Onishi, M. Takata, J. Phys. Soc. Jpn. 73, 1987 (2004).

[8] R. Lengsdorf, M. Ait-Tahar, S.S. Saxena, M. Ellerby, D.I. Khomskii, H. Micklitz, T. Lorenz, M.M. Abd-Elmeguid, Phys. Rev. B 69, 140403(R) (2004).

[9] I. Fita, R. Szymczak, R. Puzniak, I.O. Troyanchuk, J. Fink-Finowicki, Y.M. Mukovskii, V.N. Varyukin, H. Szymczak, Phys. Rev. B 71, 214404 (2005).

[10] M. Baran, V. Dyakonov, L. Gladczuk, G. Levchenko, S. Piechota, H. Szymczak, Physica C 241, 383 (1995).

[11] S. Kolesnik, B. Dabrowski, J. Mais, M. Majjiga, A. Baszczuk, unpublished. 